\title{
ORIGINAL
}

\section{CONSTRUCCIÓN DE UN ÍNDICE DE PRIVACIÓN PARA LOS BARRIOS DE MADRID Y BARCELONA (*)}

\author{
Débora Álvarez-del Arco (1,2), Marta Vicente Sánchez (3), Belén Alejos (1,2), Cruz Pascual (3) y Enrique \\ Regidor (2,3).
}

(1) Centro Nacional de Epidemiología. Instituto de Salud Carlos III.

(2) CIBER Epidemiología y Salud Pública (CIBERESP). Madrid.

(3) Departamento de Medicina Preventiva, Salud Pública e Historia de la Ciencia. Universidad Complutense de Madrid.

(*) Este estudio fue realizado en el marco del proyecto de investigación Project DEP2009-09502 "Contexto socioeconómico, disponibilidad de infraestructuras deportivas e inactividad física", financiado por el Ministerio de Ciencia e Innovación.

\begin{abstract}
RESUMEN
Fundamentos: los indicadores socioeconómicos que toman el barrio como unidad de referencia en nuestro contexto son escasos. Los objetivos de este artículo son describir el proceso de construcción y la validez de un índice de privación a nivel de barrio y analizar su asociación con la mortalidad.

Métodos: el esquema conceptual inicial del IP contuvo elementos que caracterizaban teóricamente la privación y para las que se realizó una recogida de variables de segundo nivel. El IP se adaptó a la disponibilidad de variables y a los resultados de sus análisis exploratorios. Finalmente, se realizó un análisis factorial para la validación del IP que se compuso de 5 dimensiones para Madrid (economía, población y territorio, vivienda, parque móvil y demografia) y 4 para Barcelona (las mismas salvo "demografia"). Los barrios fueron agrupados en cuartiles según la puntuación obtenida para el IP (Q4 mayor nivel de privación). Se calcularon tasas de mortalidad prematura estratificadas por sexo y ajustadas por edad y razones de mortalidad para cada cuartil.

Resultados: El IP explicó el 55\% de la variabilidad observada en los indicadores para Madrid y el $69 \%$ para Barcelona. La tasa de mortalidad prematura para el Q1 en Madrid fue 1,65 por $10^{3}$ en hombres y 0,92 por $10^{3}$ y de 2,81 por $10^{3}$ en hombres y 1,22 por $10^{3}$ en mujeres residentes en Q4. En Barcelona la tasa de mortalidad fue de 2,33 por $10^{3}$ en hombres y de 1,15 por $10^{3}$ mujeres en el Q1 y de 3,49 por $10^{3}$ en hombres y 1,52 por $10^{3}$ en mujeres del Q4.

Conclusión: Las tasas de mortalidad mostraron mayor mortalidad prematura en los barrios con un índice de privación mayor.
\end{abstract}

Palabras clave: Desigualdades en la salud. Análisis de área pequeña. Análisis factorial. Mortalidad.

\section{Correspondencia}

Débora Álvarez del Arco

Instituto de Salud Carlos III

Centro Nacional de Epidemiología

Avda. Monforte de Lemos, 5

28029 Madrid

dalvarez@isciii.es

\section{ABSTRACT \\ Process and Results of Constructing a Deprivation Index for the Districts of Madrid and Barcelona, Spain}

Background: There are few economic indicators that take the neighborhood as the unit of reference in our context. The aim of this article is to describe the process and results of secondary data collection and development of a deprivation index (DI) for the neighborhoods of the cities of Madrid and Barcelona, discussing their utility for research on health inequalities.

Methods: initial DI conceptual framework contained different elements that characterize deprivation and for which we collected secondlevel variables. ID was adapted to the availability of variables and to the results of an exploratory analysis. Finally, a factor analysis was performed to validate the IP. We built a DI based on five dimensions for Madrid (economy, population and territory, housing, cars and demographics) and 4 for Barcelona (all except "demographics"). Neighborhoods were grouped into quartiles according to their score for the DI (Q4: higher levels of deprivation). Premature mortality rates and premature mortality ratios adjusted by age were calculated for each quartile.

Results: The IP explained $55 \%$ of the observed variability in the indicators for Madrid and $69 \%$ for Barcelona. Premature mortality rate in Madrid for Q1 was 1.65 per $10^{3}$ in men and 0.92 per $10^{3}$ women and 2.81 per $10^{3}$ in men and 1.22 per $10^{3}$ in women residing in Q4. In Barcelona,the mortality rate was 2.33 per $10^{3}$ men and 1.15 per $10^{3}$ women in Q1 and 3.49 per $10^{3}$ in men and 1.52 per $10^{3}$ in women living in Q4

Conclusion: Premature mortality rates showed higher premature mortality in the most deprived districts.

Keyword: Health inequalities. Small-area analysis. Factor analysis, Statistical. Socioeconomic factors. Censuses. 


\section{INTRODUCCIÓN}

Varios estudios han puesto de manifiesto la asociación entre las características del área de residencia con la morbilidad y mortalidad observada en las personas que residenen ellas ${ }^{1-6}$. Diversas investigaciones han mostrado que el nivel socioeconómico, la configuración espacial, la cohesión social y la disponibilidad de determinados servicios en el área de residencia tienen implicaciones sobre la salud ${ }^{3}$ y los comportamientos saludables, como el consumo de alcohol o tabaco ${ }^{4}$, los hábitos alimenticios ${ }^{5} \mathrm{o}$ la práctica deportiva ${ }^{6}$.

Sin embargo, la complejidad de la conceptualización y construcción de indicadores que permitan medir el nivel socioeconómico se refleja en la heterogénea composición de los diversos índices creados para nuestro contexto ${ }^{7-9}$. Algunos autores han utilizado indicadores simples, basados en una única variable ${ }^{10}$, aunque a lo largo de los últimos años es creciente el uso de indicadores de privación multidimensionales basados en un estado de desventaja objetivable de un sujeto o grupo social con respecto a la comunidad, sociedad o nación en la que viven ${ }^{11}$. En este sentido, la privación puede ser medida utilizando determinados indicadores sociodemográficos que caracterizan a la población residente en un área $y$, también, a través de la carencia de infraestructuras urbanísticas o servicios públicos y otros recursos intangibles en el área de residencia $^{12}$. En nuestro contexto, el proyecto Medea ha sido una de las propuestas más ambiciosas en esta línea de investigación ${ }^{8}$, siendo su objetivo analizar las desigualdades socioeconómicas y medioambientales en áreas pequeñas de ciudades de España y de Europa.

En esencia, este tipo de índices tratan de categorizar cada una de las áreas en función de su posición relativa con respecto al resto, con el objetivo último de analizar la variabilidad de un problema de salud específi- co en las diversas áreas. En España se han desarrollado varias investigaciones de este tipo, relacionando las características del área de residencia con diversos resultados en términos de salud, por ejemplo, sobre la salud cardiovascular ${ }^{13}$ o la salud mental ${ }^{14}$. En nuestro contexto, la unidad de análisis más extendida es la provincia o el municipio y son escasas las investigaciones que toman el barrio como unidad de referencia.

De forma general, las hipótesis de fondo sobre las que se construyen este tipo de índices y se relacionan con resultados en salud tienen dos vertientes. Por un lado, la privación material en sentido estricto (de recursos materiales e ingresos) se relaciona de forma directa con la salud, puesto que determina de forma directa el acceso de la población a un tipo de bienes que pueden ayudar a su bienestar o a un estilo de vida saludable $^{15}$ (viviendas mejor equipadas, situadas en barrios seguros y con espacios verdes que invitan a la práctica de ejercicio físico o al desplazamiento a pie), a servicios que le pueden reportar beneficios en términos de salud (disponibilidad de servicios públicos y privados de salud, especialmente en contextos en los que la atención pública y gratuita no está garantizada, pero también de otros servicios como gimnasios) $)^{3}$ e, incluso, al acceso a determinados alimentos saludables de coste más elevado o de accesibilidad más difícil ${ }^{5}$. La otra vertiente de la privación se relaciona de forma indirecta con el acceso final a los recursos materiales y a una situación de bienestar. Estas características más indirectas se pueden medir a través de ciertos indicadores sociodemográficos, como la tasa de inmigración o la tasa de envejecimiento, que indican una cierta probabilidad de situarse en una posición económica desfavorable y, por tanto, determinan también el acceso a bienes y servicios ${ }^{8}$, aunque de forma indirecta.

En el contexto de una investigación multinivel, cuyo objetivo fue analizar la asociación entre las características de los barrios y 
los factores de riesgo de las enfermedades cardiovasculares, se elaboró un índice para medir el estado de privación.

Los objetivos de este artículo son describir el proceso de construcción y la validez de un índice de privación a nivel de barrio y analizar su asociación con la mortalidad.

\section{MATERIAL Y MÉTODOS}

Para el diseño conceptual del IP del área de residencia se realizó una revisión de la literatura científica disponible, tras la que se optó por un IP multidimensional, compuesto por 9 bloques temáticos que permitían medir aspectos diversos que caracterizaban la situación de privación en el área de residencia:

1.- Población y territorio. Las características socioeconómicas de la población y del territorio son marcadores materiales de privación (por ejemplo el nivel de estudios) que se relacionan de forma directa con la renta o los recursos disponibles.

2.- Zonas verdes y espacios ajardinados. La disponibilidad de espacios verdes está relacionada con determinadas características del área de residencia de índole material que determinan en cierta medida el bienestar de la población.

3.- Demografía: las características demográficas de la población asentada en un territorio reflejan adecuadamente la probabilidad de sufrir situaciones de mayor privación de determinadas áreas.

4.- Economía: la situación económica del área puede reflejar la escasez de recursos materiales e ingresos.

5.- Alumbrado público: la disponibilidad de ciertas infraestructuras básicas determina características materiales del área, pero también se relaciona con otros aspectos de carácter inmaterial como la percepción de seguridad.
6.- Parque móvil. El número de vehículos en el área es un marcador directo de recursos e ingresos económicos.

7.- Vivienda. Refleja el nivel de ingresos y determina, al mismo tiempo, el grado de bienestar de la población.

8.- Tráfico. Las denuncias de tráfico y los accidentes son marcadores indirectos de privación, puesto que indican el grado de accesibilidad y seguridad vial del área.

9.- Seguridad. Variables como las intervenciones de la policía son indicadores indirectos de privación puesto que reflejan el grado de conflictividad del área y de inseguridad en el que vive la población.

El esquema conceptual utilizado inicialmente se recoge en la figura 1 .

Las variables o indicadores para cada uno de los bloques temáticos del área de residencia fueron seleccionadas también de acuerdo a la revisión de la literatura sobre estudios empíricos basados en la creación de índices de privación en España ${ }^{7,9,16,17} \mathrm{y}$, aunque de forma más general, de algunos trabajos

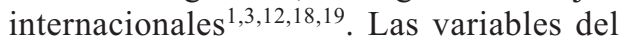
área de residencia se recogen en la tabla 1 .

La recogida de los datos secundarios para operar con las variables sobre el área de residencia para la construcción del IP se realizó entre mayo de 2010 y mayo de 2011.

Se delimitó el ámbito de la investigación a las ciudades de Madrid y de Barcelona puesto que permitían contar con una base muestral de datos individuales suficientemente amplia a nivel barrio para la siguiente fase de la investigación, cuyo objetivo era analizar la asociación entre los factores de riesgo de las enfermedades cardiovaculares y su IP.

Los datos secundarios fueron obtenidos de diversas fuentes, principalmente del cen- 
Figura 1

Esquema conceptual: bloques temáticos teóricos que conforman el Î́ndice de Privación

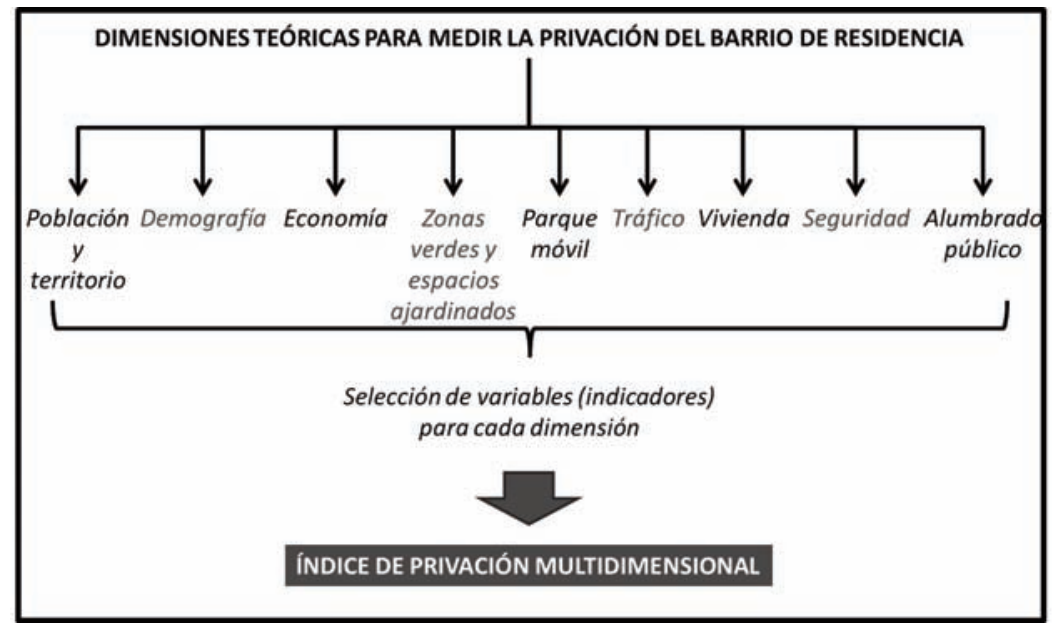

so de Población y Viviendas ${ }^{20}$, del Padrón Municipal de Habitantes ${ }^{21}$ y de los anuarios estadísticos $^{22,23}$ y otros bancos de datos ${ }^{24}$ elaborados por los servicios de estadística de los ayuntamientos: Departament d'Estadística (Ayuntamiento de Barcelona) y Dirección General de Estadística (Ayuntamiento de Madrid). Además se contactó con diversas instituciones municipales y otras entidades para solicitar la información necesaria sobre las variables descritas anteriormente desagregadas a nivel barrio. Las fuentes de información y las instituciones contactadas para la solicitud de las variables de cada área temática se recogen en la tabla 1 .

La recogida de datos de segundo nivel para las ciudades de Madrid y Barcelona planteó principalmente dos problemas: la información desagregada a nivel de barrio no estaba disponible para muchas de las variables seleccionadas y, además, los indicadores de ambas ciudades en muchos casos no se correspondían, puesto que las unidades de medida utilizadas eran distintas y no equiparables entre sí. Por ello hubo que reelaborar el esquema conceptual definitivo para construir el IP, el cual se recoge en la figura 2 .
Finalmente, se diseñó un İP basado en cinco dimensiones: a) población y territorio; b) economía; c) vivienda; d) parque móvil y e) demografía.

Para la construcción y validación del índice fueron realizados análisis factoriales. En base a la experiencia de DomínguezBerjón et al. ${ }^{25} \mathrm{y}$ al procedimiento diseñado por Carr-Hill y Chalmers Dixon ${ }^{26}$, la construcción del índice se realizó en tres etapas:

1. Selección de las variables (tabla 2). En primer lugar se seleccionaron las variables a incluir para cada una de las dimensiones teóricas del IP en base al esquema conceptual definitivo. Se realizó un análisis descriptivo exploratorio para cada una de las variables preliminares, valorando si se cumplían los supuestos paramétricos de distribución normal de las variables utilizando el estadístico Shapiro-Wilk. De las 12 variables seleccionadas para el IP, sobre los barrios de Madrid presentaron ausencia de normalidad 10 variables y 3 sobre los barrios de Barcelona. La tabla 2 resume los resultados de esta fase para cada variable y cada ciudad. 
Tabla 1

\section{Variables inicialmente seleccionadas en función de la dimensión teórica de la privación en la que se engloba y fuentes de información o entidades contactadas.}

\begin{tabular}{|c|c|c|}
\hline Población y territorio & $\begin{array}{l}\text { Porcentaje población de analfabetos + sin estudios; porcentaje población } \\
\text { con estudios de tercer grado; Superficie; población; superficie de calza- } \\
\text { das; superficie de aceras; superficie de pasos de peatones; superficie de } \\
\text { pasos de carruajes; superficie de superficies especiales; longitud de bordi- } \\
\text { llos; longitud de ejes de calzada; longitud de calles; número de calles; su- } \\
\text { perficie de zonas y calles con prioridad a viandantes }\end{array}$ & $\begin{array}{l}\text { Instituto Nacional de Estadístical; Departament d'Estadística }{ }^{\mathrm{II}} \text {; Dirección Gene- } \\
\text { ral de Estadística } \\
\text { de Cartografia i A Área de Medi Loca }^{\mathrm{IIV}} \text {; Área de Gobierno de Obras y Espacios Públicos }{ }^{\mathrm{III}} \text {; } \\
\text { Dirección General de Urbanismo y Estrategia TerritorialV }\end{array}$ \\
\hline Zonas verdes y espacios ajardinados & $\begin{array}{l}\text { Arbolado; } \mathrm{m}^{2} \text { de superficie verde urbana; superficie de parques urbanos; } \\
\text { número de jardineras }\end{array}$ & $\begin{array}{l}\text { Institut Municipal de Parcs i JardinsII; Área de Gobierno de Medio Ambiente, } \\
\text { Seguridad y Movilidad"II. }\end{array}$ \\
\hline Demografía & Crecimiento migratorio; tasa inmigración & Departament d'Estadística ${ }^{\mathrm{II}}$; Dirección General de Estadística ${ }^{\mathrm{III}}$. \\
\hline Economía & $\begin{array}{l}\text { Tasa de Paro; Tasa de actividad; Comercio al por mayor; comercio al por } \\
\text { menor; número de locales dedicados al comercio; número de locales de } \\
\text { comercio ocupados }\end{array}$ & $\begin{array}{l}\text { Instituto Nacional de Estadística }{ }^{\mathrm{I}} \text {; Departament d'Estadística }{ }^{\mathrm{II}} \text {; Observatori } \\
\text { Econòmic Local }^{\mathrm{IV}} \text {; Cambra de comerç de Barcelona; Dirección General de Esta- } \\
\text { dística }^{\mathrm{III}} \text {. }\end{array}$ \\
\hline Alumbrado público & $\begin{array}{l}\text { Puntos luz alumbrado viario; potencia de energía total; consumo de ener- } \\
\text { gía; lámparas alumbrado viario; lámparas alumbrado artístico }\end{array}$ & $\begin{array}{l}\text { Àrea de Medi Ambient i Serveis Urbans }{ }^{\mathrm{II}} \text {; Centre de Recursos Barcelona Soste- } \\
\text { nible }{ }^{\mathrm{II}} \text {; Servicios de Energía y Calidad ambiental }{ }^{\mathrm{II}} \text {; Área de Gobierno de Obras y } \\
\text { Espacios Públicos }{ }^{\mathrm{II}} \text {. }\end{array}$ \\
\hline Parque móvil & $\begin{array}{l}\text { Porcentaje población que dispone sólo de un vehículo; porcentaje pobla- } \\
\text { ción que dispone más de un vehículo; número de automóviles; }\end{array}$ & $\begin{array}{l}\text { Instituto Nacional de Estadística'; Departament d'Estadística"II Dirección Gene- } \\
\text { ral de Estadísticall. }\end{array}$ \\
\hline Vivienda & $\begin{array}{l}\text { Número medio de habitaciones; Superficie media de la vivienda;Porcen- } \\
\text { taje de viviendas sin calefacción; Porcentaje de población en viviendas sin } \\
\text { calefacción; Porcentaje de población en vivienda de alquiler. }\end{array}$ & $\begin{array}{l}\text { Instituto Nacional de Estadística'; Institut Municipal d'Hisenda }{ }^{\mathrm{II}} \text {; Gerencia Re- } \\
\text { gional del Catastro de Cataluña } a^{\mathrm{VI}} \text {; Secretaría de Estado de Vivienda y Actuacio- } \\
\text { nes Urbanas }{ }^{\mathrm{VII}} \text {; Patronat Municipal de l'Habitatge }{ }^{\mathrm{II}} \text {; Gerencia Regional del Ca- } \\
{\text { tastro de Madrid }{ }^{\mathrm{VI}} \text {; Área de Gobierno de Urbanismo y Vivienda }}^{\mathrm{III}} \text {; Instituto de } \\
\text { Vivienda de Madrid } \text {; Dirección General de Vivienda y Rehabilitación }{ }^{\mathrm{V}} \text {; Tecni- } \\
\text { grama SA (Agencia privada); Facilisimo Interactive SL (Agencia privada); So- } \\
\text { ciedad de Tasación SA (Agencia privada). }\end{array}$ \\
\hline Tráfico & Denuncias de infracciones de circulación; accidentes de tráfico & $\begin{array}{l}\text { Gerència de Prevenció, Seguretat i Mobilitat" }{ }^{\mathrm{II}} \text {; Guàrdia Urbana de Barcelona }{ }^{\mathrm{II}} \text {; } \\
\text { Área de Gobierno de Seguridad y Servicios a la ComunidadIII; Dirección Gene- } \\
\text { ral de Tráfico }\end{array}$ \\
\hline Seguridad & $\begin{array}{l}\text { Intervenciones de la policía municipal con detenidos e imputados; inter- } \\
\text { venciones operativas; intervenciones no operativas; delitos instruidos; fal- } \\
\text { tas administrativas instruidas; faltas penales instruidas; órdenes judiciales } \\
\text { instruidas; otras faltas instruidas; delitos y faltas localizados }\end{array}$ & $\begin{array}{l}\text { Guàrdia Urbana de Barcelona }{ }^{\mathrm{II}} \text {; Dirección General de Policía }{ }^{\mathrm{VIII}} \text {; Mossos d'es- } \\
\text { quadraIX ; Área de Gobierno de Seguridad y Servicios a la Comunidad }{ }^{\mathrm{III}} \text {; Poli- } \\
\text { cía Municipal de Madrid }{ }^{\mathrm{IV}} \text {. }\end{array}$ \\
\hline
\end{tabular}

${ }^{\text {I }}$ Ministerio de Economía y Competitividad; ${ }^{\text {II }}$ Ayuntamiento de Barcelona; ${ }^{\text {III }}$; Ayuntamiento de Madrid; ${ }^{\text {IV }}$ Diputación de Barcelona; ${ }^{\text {V }}$ Comunidad de Madrid; ${ }^{\text {VI }}$ Ministerio

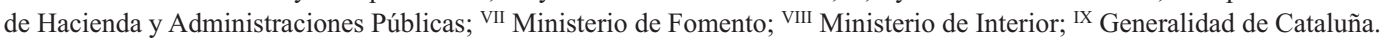


Figura 2

Esquema conceptual definitivo para la construcción del Índice de Privación

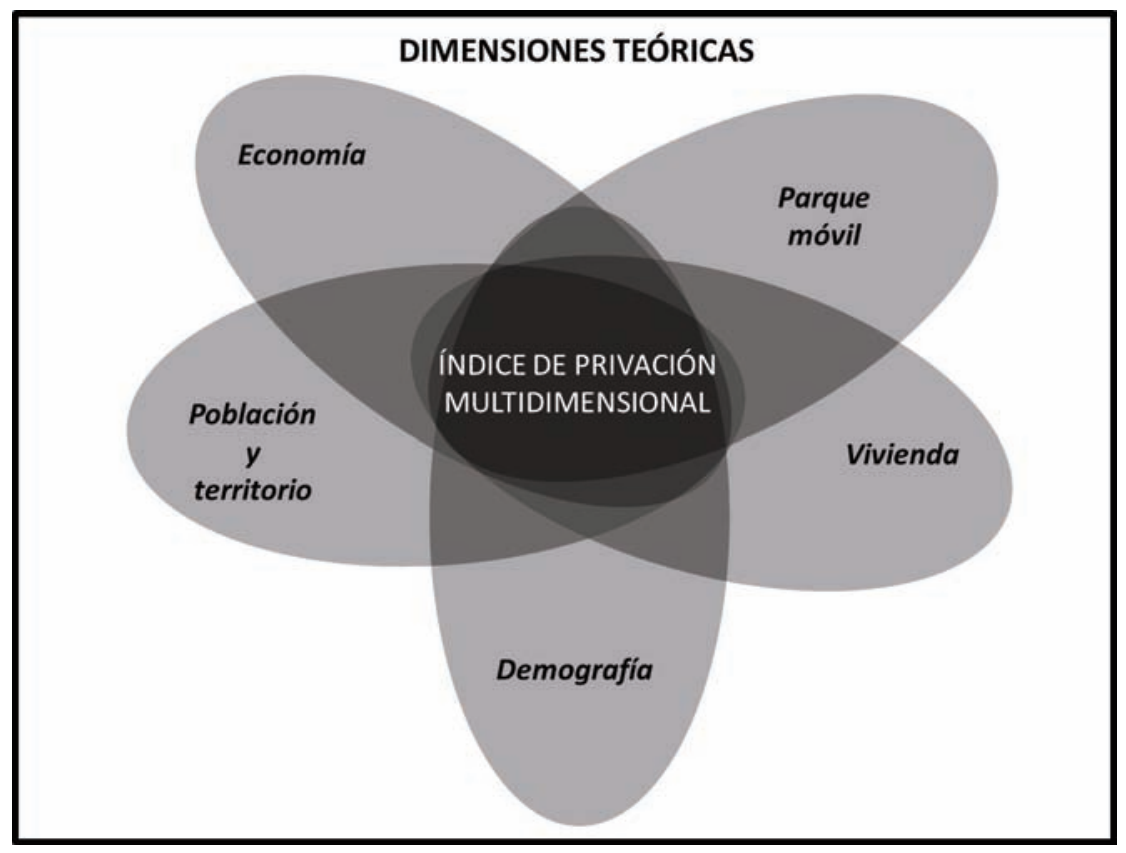

2. Validación de las variables. En segundo lugar, cada una de las variables fue asignada a la dimensión correspondiente del IP. En la tabla 3 se describen las variables utilizadas para la construcción de cada una de las dimensiones que compusieron el IP, así como las fuentes de las que se obtuvieron dichas variables. Utilizando el coeficiente de correlación lineal de Pearsons se comprobó la independencia lineal de las variables, para evitar problemas de colinealidad. Además se realizó un análisis factorial exploratorio y se analizaron las comunalidades que representan el porcentaje de la variabilidad de cada variable que es explicada por los factores comunes a todas las variables.

En esta fase se observaron problemas de multicolinealidad entre variables de dos de las dimensiones de análisis:

a) En la dimensión Vivienda se descartaron tres de las cuatro variables inicialmente propuestas quedando únicamente "porcentaje de viviendas sin calefacción". La variable "número medio de habitaciones" fue descartada puesto que presentó una comunalidad no válida.

b) En la dimensión "Parque móvil" se decidió elegir la variable "porcentaje de la población que posee sólo un vehículo".

3. Validación del ÍP. De acuerdo a los resultados de las dos fases previas, se realizó un análisis factorial para la construcción y validación del ÍP, optando por el uso de metodología multivariante, de modo que el peso de cada factor estuviera determinado por las relaciones estadísticas entre las variables dentro del área geográfica seleccionada ${ }^{8}$. Para el análisis de factores comunes se utilizó el método de factorización de ejes principales para distribuciones no-normales ${ }^{27}$.

En esta fase se valoró la adecuación del análisis factorial para la construcción de este índice, comprobando el ajuste de la muestra mediante el Coeficiente Kaiser-Meyer-Olkin 
Tabla 2

Resultados del análisis previo de las variables a incluir

\begin{tabular}{|c|c|c|c|c|c|c|c|c|}
\hline \multirow{2}{*}{ Variables } & \multicolumn{2}{|c|}{ Normalidad } & \multicolumn{2}{|c|}{ Asociación lineal } & \multicolumn{2}{|c|}{$\begin{array}{c}\text { Otros } \\
\text { resultados }\end{array}$} & \multicolumn{2}{|c|}{ Variables incluidas } \\
\hline & Madrid & Barcelona & Madrid & Barcelona & Madrid & Barcelona & Madrid & Barcelona \\
\hline Tasa de Paro & $\sqrt{ }$ & $\sqrt{ }$ & $\sqrt{ }$ & $\sqrt{ }$ & - & - & $\sqrt{ }$ & $\sqrt{ }$ \\
\hline Porcentaje población de analfabetos + sin estudios & $\mathrm{X}$ & $\sqrt{ }$ & $\sqrt{ }$ & $\sqrt{ }$ & - & - & $\sqrt{ }$ & $\sqrt{ }$ \\
\hline Número medio de habitaciones & $\mathrm{X}$ & $\mathrm{X}$ & $\sqrt{ }$ & $\sqrt{ }$ & Comunalidad $>1$ & & $\mathrm{X}$ & $\sqrt{ }$ \\
\hline Superficie media de la vivienda & $\mathrm{X}$ & $\sqrt{ }$ & $\sqrt{ }$ & $\sqrt{ }$ & - & - & $\sqrt{ }$ & $\sqrt{ }$ \\
\hline$\%$ de viviendas sin calefacción & $\mathrm{X}$ & $\sqrt{ }$ & $\sqrt{ }$ & $\sqrt{ }$ & - & - & $\sqrt{ }$ & $\sqrt{ }$ \\
\hline$\%$ de población en vivienda sin calefacción & $\mathrm{X}$ & $\sqrt{ }$ & $\sqrt{ }$ & $\bar{X}$ & Colinealidad $(*)$ & Colinealidad $(*)$ & $\bar{X}$ & $\mathrm{X}$ \\
\hline$\%$ de población en vivienda de alquiler & $\mathrm{X}$ & $\bar{X}$ & $\mathrm{X}$ & $\sqrt{ }$ & Colinealidad $(*)$ & Colinealidad $(*)$ & $\mathrm{X}$ & $\sqrt{ }$ \\
\hline Precio de vivienda de segunda mano & $\mathrm{X}$ & $\sqrt{ }$ & $\sqrt{ }$ & $\sqrt{ }$ & - & - & $\sqrt{ }$ & $\sqrt{ }$ \\
\hline \% población que dispone un vehículo & $\mathrm{X}$ & $\sqrt{ }$ & $\sqrt{ }$ & $\sqrt{ }$ & Colinealidad $(* *)$ & Colinealidad (**) & $\sqrt{ }$ & $\sqrt{ }$ \\
\hline$\%$ población que dispone más de un vehículo & $\mathrm{X}$ & $\sqrt{ }$ & $\sqrt{ }$ & $\sqrt{ }$ & Colinealidad $(* *)$ & Colinealidad $(* *)$ & $\mathrm{X}$ & $\mathrm{X}$ \\
\hline Número de turismos & $\sqrt{ }$ & $\sqrt{ }$ & $\mathrm{X}$ & $\mathrm{X}$ & - & - & $\mathrm{X}$ & $\mathrm{X}$ \\
\hline Tasa de inmigración & $\mathrm{X}$ & $\mathrm{X}$ & $\sqrt{ }$ & $\mathrm{X}$ & - & - & $\sqrt{ }$ & $\mathrm{X}$ \\
\hline
\end{tabular}

$\sqrt{ }$ : Cumple criterio o se incluye variable. X: No cumple criterio o no se incluye variable. Comunalidad $>1$ : valor de la comunalidad no válido, se descarta la variable. Colinealidad (*): las variables presentan colinealidad entre sí. Colinealidad (**): las variables presentan colinealidad entre sí. 
Tabla 3

Variables utilizadas para cada indicador y fuente de datos

\begin{tabular}{|c|c|c|}
\hline Dimensión & Variables & Fuente \\
\hline Economía & Tasa de paro o tasa de actividad & Censo Población y Viviendas (2001) \\
\hline Población y territorio & $\begin{array}{l}\text { Porcentaje población de analfabetos }+ \text { sin estudios } \\
\text { o con estudios de tercer grado }\end{array}$ & Censo Población y Viviendas (2001) \\
\hline \multirow{6}{*}{ Vivienda } & Número medio de habitaciones & \multirow{5}{*}{ Censo Población y Viviendas (2001) } \\
\hline & Superficie media de la vivienda & \\
\hline & Porcentaje de viviendas sin calefacción & \\
\hline & Porcentaje de población en viviendas sin calefacción & \\
\hline & Porcentaje de población en vivienda de alquiler & \\
\hline & Precio de vivienda de segunda mano & Anuarios estadísticos municipales (2007) \\
\hline \multirow{3}{*}{ Parque móvil } & Porcentaje población que dispone sólo de un vehículo & \multirow{3}{*}{ Censo Población y Viviendas (2001) } \\
\hline & Porcentaje población que dispone más de un vehículo & \\
\hline & Número de automóviles & \\
\hline Demografía & Tasa de inmigración & Padrón municipal de habitantes (2007) \\
\hline
\end{tabular}

(KMO), que contrasta la magnitud de las correlaciones parciales entre las variables, y se aplicó la prueba de esfericidad de Bartlett para comprobar que el modelo factorial construido fuese adecuado para explicar los datos de la muestra. La ausencia de normalidad de buena parte de las variables seleccionadas derivó en la extracción de factores mediante el método de ejes principales. Finalmente, se construyó un IP compuesto por cinco dimensiones para Madrid (economía, población y territorio, vivienda, parque móvil y demografía) y cuatro para Barcelona. (economía, población y territorio, vivienda, parque móvil). Puesto que los indicadores que componían el índice para cada ciudad fueron distintos, los resultados no son comparables entre ambas urbes. Se analizaron las saturaciones de los modelos para valorar la correlación del factor con el resto de las variables y se analizaron los porcentajes de variabilidad explicada por el índice. Todos los cálculos fueron realizados utilizando el programa estadístico SPSS ${ }^{18}$.

Los barrios fueron agrupados en cuartiles en función de las puntuaciones que obtuvieron en el IP: el cuartil 1 (Q1) agrupó los barrios con menor grado de privación y el cuartil 4 (Q4) los barrios con mayor nivel de privación.
Por último, con objeto de evaluar la validez de este índice, se calcularon las tasas de mortalidad prematura (16 a 64 años) estratificadas por sexo y ajustadas por edad y las razones de mortalidad del periodo 20082010 para cada uno de los cuartiles en los que se agruparon los barrios.

\section{RESULTADOS}

El ÍP explicó el 55,05\% de la variabilidad observada en los indicadores para Madrid y el $69,20 \%$ para Barcelona, tal y como muestra la tabla 4. Las variables que compusieron este índice presentaron saturaciones que se situaron por encima de 0,6 .

Los valores medios de cada una de las variables que conformaron el índice para cada cuartil y para cada ciudad se recogen en la tabla 4. La tasa de paro se situó en Madrid en el 9,7\% para los barrios con menor privación relativa y en el $14,6 \%$ en los barrios con mayor nivel de privación, siendo estas puntuaciones muy cercanas a las que se observaron en Barcelona para los barrios de los mismos cuartiles ( $9 \%$ y $15,1 \%$, respectivamente). Del mismo modo, se observaron diferencias sustantivas en la proporción de personas analfabetas o sin estudios entre los barrios 


\section{Tabla 4}

Dimensiones, indicadores, saturaciones, variabilidad explicada por el índice de privación y valor medio de cada uno de los indicadores para cada cuartil (Q) de Madrid y Barcelona

\begin{tabular}{|c|c|c|c|c|c|c|c|c|c|c|}
\hline \multirow{3}{*}{ Dimensión (indicador) } & \multicolumn{5}{|c|}{ Madrid } & \multicolumn{5}{|c|}{ Barcelona } \\
\hline & \multirow[b]{2}{*}{ Saturaciones } & \multicolumn{4}{|c|}{ Valor medio } & \multirow[b]{2}{*}{ Saturaciones } & \multicolumn{4}{|c|}{ Valor medio } \\
\hline & & $\begin{array}{l}\text { Q1 } \\
\%\end{array}$ & $\begin{array}{l}\text { Q2 } \\
\%\end{array}$ & $\begin{array}{l}\text { Q3 } \\
\%\end{array}$ & $\begin{array}{l}\text { Q4 } \\
\%\end{array}$ & & $\begin{array}{l}\text { Q1 } \\
\%\end{array}$ & $\begin{array}{l}\mathrm{Q} 2 \\
\%\end{array}$ & $\begin{array}{l}\text { Q3 } \\
\%\end{array}$ & $\begin{array}{l}\text { Q4 } \\
\%\end{array}$ \\
\hline Economía (tasa de paro) & 0,67 & 9,7 & 11,3 & 13,2 & 14,6 & 0,79 & 9,0 & 10,3 & 11,8 & 15,1 \\
\hline $\begin{array}{l}\text { Población y territorio } \\
(\% \text { de población analfabetos }+ \text { sin estudios })\end{array}$ & 0,62 & 5,0 & 8,6 & 15,8 & 19,3 & 0,80 & 6,2 & 10,8 & 16,2 & 21,6 \\
\hline $\begin{array}{l}\text { Vivienda } \\
\text { (\% de población sin calefacción) }\end{array}$ & 0,82 & 5,1 & 12,2 & 21,0 & 34,6 & 0,95 & 27,6 & 46,8 & 54,7 & 68,3 \\
\hline $\begin{array}{l}\text { Parque móvil } \\
\text { (\% de población que dispone sólo de un vehículo) }\end{array}$ & 0,84 & 56,7 & 76,8 & 81,1 & 85,8 & 0,77 & 77,0 & 86,6 & 88,3 & 90,9 \\
\hline $\begin{array}{l}\text { Demografía } \\
\text { (Tasa de inmigración) }\end{array}$ & 0,74 & 6,9 & 10,1 & 16,0 & 21,3 & $(*)$ & - & - & - & - \\
\hline Variabilidad explicada & 55,05 & & & & & 69,20 & & & & \\
\hline
\end{tabular}

(*) Debido a la ausencia de asociación lineal entre la tasa de inmigración y el resto de variables, finalmente no fue incluida en el análisis. Q1: Barrios con un menor IP; Q4: Barrios con un mayor IP.

categorizados en el Q1 (5\% en Madrid y $6,2 \%$ en Barcelona, con respecto a aquéllos categorizados en el Q4 (19,3\% en Madrid y $21,6 \%$ en Barcelona).
La figura 3 muestra la categorización en cuartiles de los barrios de Madrid y Barcelona en función de su IP.

Figura 3

Distribución geográfica del Índice de Privación en los barrios de Madrid y Barcelona

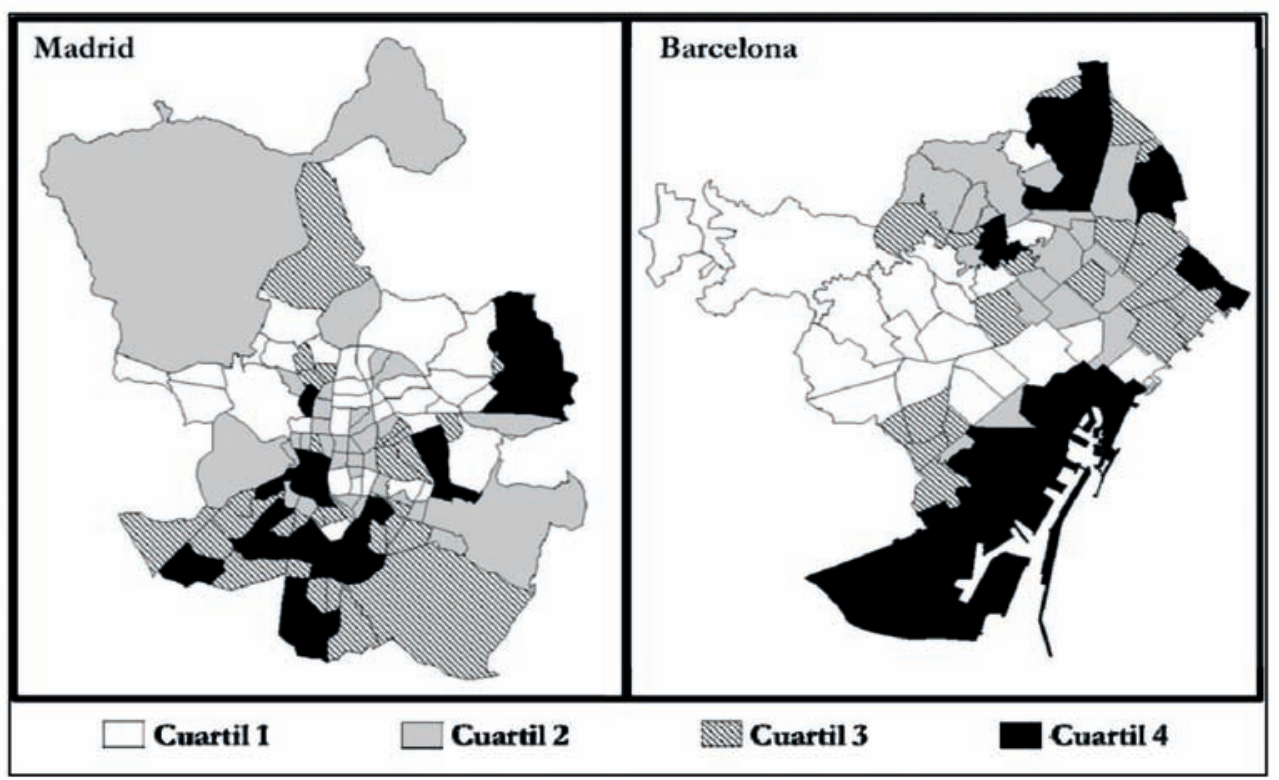


Tabla 5

Tasa de mortalidad prematura (16 a 64 años) ajustada por edad por $10^{3}$ habitantes y razón de tasas según el índice de privación para hombres y mujeres en Madrid y en Barcelona. Período 2008-2010

\begin{tabular}{|c|c|c|c|c|c|c|c|c|}
\hline \multirow{2}{*}{ Cuartil } & \multicolumn{2}{|c|}{$\begin{array}{c}\text { Tasa de mortalidad prematura (16 a 64 años) } \\
\text { ajustada por edad por } 10^{3} \text { habitantes }\end{array}$} & \multicolumn{4}{|c|}{$\begin{array}{c}\text { Razón de tasas } \\
\text { de mortalidad }\end{array}$} \\
\cline { 2 - 9 } & \multicolumn{2}{|c|}{ Madrid } & \multicolumn{2}{|c|}{ Barcelona } & \multicolumn{2}{|c|}{ Madrid } & \multicolumn{2}{c|}{ Barcelona } \\
\cline { 2 - 9 } & Hombres & Mujeres & Hombres & Mujeres & Hombres & Mujeres & Hombres & Mujeres \\
\hline Q1 & 1,65 & 0,92 & 2,33 & 1,15 & 1,00 & 1,00 & 1,00 & 1,00 \\
\hline Q2 & 2,19 & 1,06 & 2,57 & 1,19 & 1,33 & 1,15 & 1,10 & 1,04 \\
\hline Q3 & 2,44 & 113 & 2,82 & 1,35 & 148 & 1,22 & 1,21 & 1,18 \\
\hline Q4 & 2,81 & 1,22 & 3,49 & 1,52 & 1,70 & 133 & 1,50 & 1,32 \\
\hline
\end{tabular}

La tabla 5 muestra las tasas de mortalidad prematura estratificadas por sexo y ajustadas por edad y las razones de mortalidad para cada uno de los cuartiles en los que se distribuyen los barrios en función de la puntuación obtenida en el IP. Se observó una relación directa entre las tasas de mortalidad y el IP del área para ambos sexos. La razón de mortalidad aumentó a medida que aumentó el cuartil: en los barrios de mayor privación, la mortalidad prematura fue también mayor. En los barrios del Q4 (más deprimidos) de Madrid, la tasa mortalidad de mortalidad prematura en hombres fue 1,7 veces mayor que en los del Q1, y en mujeres 1,33 veces mayor. En Barcelona fue 1,5 veces mayor en hombres y 1,32 en mujeres en los barrios del Q4 que en los barrios agrupados en el Q1 según la puntuación obtenida en el IP.

\section{DISCUSIÓN}

El Índice de Privación construido es válido para medir el nivel de privación de las áreas. La proporción de variabilidad explicada por el índice para cada ciudad se asemeja a lo observado en otras investigaciones similares. Henson et al. ${ }^{28}$, en su revisión de 60 análisis factoriales, mostraron que la proporción media de varianza explicada por los factores fue de un $52 \%$. En este sentido, a pesar de incluir un número menor de factores, nuestro IP se ajusta mejor a los barrios de la ciudad de Barcelona que a los de la ciudad de Madrid. La mejor adecuación del índice a la ciudad de Barcelona puede deberse, en gran medida, a la reorganización administrativa de la ciudad puesta en marcha en $2007^{29}$ que la dividió en barrios creados en base a criterios sociológicos. Los barrios de Madrid, sin embargo, se corresponden a divisiones administrativas de la ciudad $y$, aunque se trata de unidades con cierta homogeneidad interna, no son constructos elaborados para agrupar áreas conexas de características similares.

Por otra parte, el índice propuesto refleja las diferencias de mortalidad prematura en hombres y en mujeres entre las distintas áreas según su categorización en los distintos cuartiles del IP, tal y como muestra la tasa de mortalidad prematura. En los barrios del cuartil más deprimido, la tasa de mortalidad es sensiblemente mayor que en los barrios del cuartil con menor puntuación en el Índice de Privación. En una segunda fase, se valorará la existencia de asociación entre el nivel de privación del área y los resultados en términos de factores de riesgo para la salud cardiovascular de la población residente en las ciudades de Madrid y Barcelona. Varios autores han señalado para otros contextos 
que las personas residentes en los barrios con mayor nivel de privación sufren mayores tasas de enfermedades isquémicas o infartos ${ }^{3,15,30}$, aislando el efecto de las características individuales de las personas. En este sentido, los modelos multinivel son el mejor abordaje para distinguir el efecto de las características contextuales del efecto de las características individuales $6,10,31-33$.

El IP descrito aporta información desagregada a nivel de barrio, lo que supone una novedad con respecto a otros índices construidos para nuestro contexto, como el del Proyecto MEDEA ${ }^{8}$. Investigaciones realizadas en otros países han mostrado que el barrio es la unidad que mejor resume las características y con la que interactúan los sujetos, debido fundamentalmente a su tamaño y configuración interna $^{32}$. A pesar de ello, las investigaciones realizadas en nuestro país que toman el barrio como unidad de referencia son muy escasas, lo que en muchos casos está relacionado con la falta de disponibilidad de datos desagregados a nivel de barrio ${ }^{34}$.

Sin embargo, el IP elaborado presenta algunas limitaciones. En primer lugar, en la construcción del índice han sido utilizados únicamente indicadores objetivos, mientras que otras investigaciones tienen en cuenta medidas de privación subjetivas, basadas en las percepciones de los habitantes de las áreas ${ }^{35}$. Por otra parte, la diferente construcción del índice para cada una de las ciudades hace más compleja la siguiente fase de la investigación, puesto que no permitirá comparar ambas ciudades. Además, el índice diseñado mide la privación a nivel del barrio de residencia pero no permite la medición acumulativa de la privación que experimentan los individuos de dicho barrio, que se exponen no sólo a las características del área en la que residen si no también a las de otras áreas, por ejemplo el lugar en el que desarrollan su actividad laboral y, por tanto, no permite la medición de la privación múltiple en sentido estricto.

Por otra parte, a pesar de la utilidad de este tipo de índices, la recogida de datos de segundo nivel planteó una serie de problemas ligados a la carencia de indicadores desglosados a nivel barrio y a la falta de homogeneidad de indicadores comunes para las ciudades de Madrid y Barcelona, puesta ya de manifiesto para nuestro contexto por otras investigaciones ${ }^{34}$. El contacto con las diversas instituciones para la obtención de datos fue un proceso largo y complejo y en muchos casos infructuoso. Finalmente se recurrió a la principal fuente de datos secundarios de España: el Censo de Población y Viviendas que, si bien proporciona datos para el conjunto del país desagregados a nivel de sección censal, ofrece información socioeconómica que puede resultar insuficiente para los objetivos de la investigación y además, ser anacrónica, puesto que se utilizó la información de la Edición de $2001^{20}$. Sería muy adecuado replicar la construcción del IP cuando esté disponible la información completa del Censo de Población y Viviendas de 2011, con el fin de valorar si se han producido cambios relevantes en las características de los barrios entre ambos períodos. Adicionalmente, el esquema conceptual en el que se basó inicialmente la construcción del IP tuvo que ser modificado en función de la disponibilidad de variables y hubo que adaptarlo a dicha disponibilidad, reduciéndose el número de dimensiones que finalmente lo compusieron.

De forma global, se considera que la construcción de un índice de privación es un buen mecanismo para medir de forma sintética las desigualdades socioeconómicas entre áreas y parece que podría ser útil para valorar desigualdades de salud en dichas áreas, tal y como muestran las razones de mortalidad prematura. Sin 
embargo, es necesario favorecer e incentivar la existencia y accesibilidad de fuentes adecuadas de información con un grado de desagregación geográfica suficiente que permita desarrollar investigaciones dirigidas a medir el impacto de las características del área de residencia en la salud.

\section{AGRADECIMIENTOS}

Al Instituto Nacional de Estadística, a la Dirección General de Estadística del Ayuntamiento de Madrid, al Departament d'Estadística del Ayuntamiento de Barcelona y a las diversas instituciones municipales contactadas, por su ayuda a lo largo de todo el proceso de recogida de datos secundarios.

\section{BIBLIOGRAFÍA}

1. Diez Roux AV. Investigating neighborhood and area effects on health. Am J Public Health. 2001;91:1783-9.

2. Wen M, Browning CR, Cagney KA. Poverty, affluence, and income inequality: neighborhood economic structure and its implications for health. Soc Sci Med. 2003;57:843-60.

3. Sundquist K, Malmstrom M, Johansson SE. Neighbourhood deprivation and incidence of coronary heart disease: a multilevel study of 2.6 million women and men in Sweden. J Epidemiol Community Health. 2004;58:71-7.

4. Lin EY, Witten K, Casswell S, You RQ. Neighbourhood matters: Perceptions of neighbourhood cohesiveness and associations with alcohol, cannabis and tobacco use. Drug Alcohol Rev. 2012 Jun;31(4):40212.

5. Franco M, Diez Roux AV, Glass TA, Caballero B, Brancati FL. Neighborhood characteristics and availability of healthy foods in Baltimore. Am J Prev Med. 2008;35:561-7.

6. Pascual C, Regidor E, Astasio P, Ortega P, Navarro $\mathrm{P}$, Dominguez V. The association of current and sustained area-based adverse socioeconomic environment with physical inactivity. Soc Sci Med. 2007;65:45466.

7. Benach J, Yasui Y. Geographical patterns of excess mortality in Spain explained by two indices of deprivation. J Epidemiol Community Health. 1999;53:423-31.
8. Dominguez-Berjon MF, Borrell C, Cano-Serral G, Esnaola S, Nolasco A, Pasarin MI, Ramis R, Saurina C, Escolar-Pujolar A. [Constructing a deprivation index based on census data in large Spanish cities(the MEDEA project)]. Gac Sanit. 2008;22:179-87.

9. Lertxundi-Manterola A., Saurina C., Sáez M., OcañaRiola R. Construcción de un índice de privación material para los municipios de la Región Sanitaria de Girona. Estudios de Economía Aplicada. 2005; 23: 331-353.

10. Pascual C, Regidor E, Gutierrez-Fisac JL, Martinez D, Calle ME, Dominguez V. Bienestar material de la provincia de residencia e inactividad física. Gac Sanit. 2005;19:424-32.

11. Townsend P. Deprivation. J Soc Policy. 1987;16, 125-146.

12. Social Disadvantadge Research Centre (SDRC). Scottish indices of deprivation 2003. Oxford: SDRC;2003.

13. Dominguez-Berjon MF, Gandarillas A, Segura del PJ, Zorrilla B, Soto MJ, Lopez L, Duque I, Marta MI, Abad I. Census tract socioeconomic and physical environment and cardiovascular mortality in the Region of Madrid (Spain). J Epidemiol Community Health. 2010;64:1086-93.

14. Jarrin I, Garcia-Fulgueiras A, Ibanez-Rojo V, Alvarez D, Garcia-Pina R, Fernandez-Liria A, Garcia-Ortuzar V, Diaz D, Rodriguez-Arenas MA, Mazarrasa L, Zunzunegui MV, Llacer A, Del AJ. Absence of protective ethnic density effect on Ecuadorian migrants' mental health in a recent migration setting: a multilevel analysis. Soc Psychiatry Psychiatr Epidemiol. 2013 Jan;48(1):95-103.

15. Diez Roux AV, Merkin SS, Arnett D, Chambless L, Massing M, Nieto FJ, Sorlie P, Szklo M, Tyroler HA, Watson RL. Neighborhood of residence and incidence of coronary heart disease. N Engl J Med. 2001;345:99106.

16. Arias A., Rebagliato M., Palumbo M.A., Bellver R., Ashton J., Colomer C. et al. Desigualdades en salud en Barcelona y Valencia. Med Clín (Barc). 1993; 100: 281287.

17. Dominguez-Berjon MF, Borrell C, Benach J, Pasarin MI. Medidas de privación material en los estudios de áreas geográficas pequeñas. Gac Sanit. 2001; 15: 23-33.

18. Jordan H, Roderick P, Martin D. The Index of Multiple Deprivation 2000 and accessibility effects on health. J Epidemiol Community Health. 2004;58:250-7. 
19. Townsend P., Phillimore P., Beattie A. Health and deprivation. Inequality and the North. London: Routledge; 1988 .

20. Censo de Población y Viviendas 2001. Instituto Nacional de Estadística (INE). [citado 15 de Julio de $20013]$. Disponible en: http://www.ine.es/jaxi/menu.do?type=pcaxis \&path= $\% 2 \mathrm{Ft} 20 \% 2 \mathrm{Fe} 242 \&$ file $=$ inebase $\& \mathrm{~L}=0$.

21. Padrón Municipal de habitantes. Instituto Nacional de Estadística (INE). [citado 15 Julio de 2013]. D i s p on ible http://www.ine.es/jaxi/menu.do?type=pcaxis \&path= $\% 2 \mathrm{Ft} 20 \% 2 \mathrm{Fe} 245 \&$ file $=$ inebase $\& \mathrm{~L}=0$.

22. Anuario Estadístico Municipal de la ciudad de Madrid. Ayuntamiento de Madrid. Departamento de Estadística. [citado 15 de Julio de 2013]. Disponible en: http://www.madrid.es/portales/munimadrid/es/Inicio/Ayuntamiento/Estadistica/Publicaciones.

23. Anuari Estadístic de la Ciutat de Barcelona. Ajuntament de Barcelona.Departament de Estadistica. [citado 15 de Julio de 2013]. Disponible en: http://www.bcn.cat/estadistica/catala/dades/.

24. OpenData BCN. Ajuntament de Barcelona.Servei de dades obertes. [citado 15 de Julio de2013]. Disponible en: http://w20.ben.cat/opendata/ODABCN.aspx?lang=CAS.

25. Dominguez-Berjon MF, Borrell C, Pastor V. [Small area-based socio-economic indicators in the study of inequalities in health]. Gac Sanit. 2004;18:92100 .

26. The Public Health Observatory Handbook of Health Inequalities Measurement. South East Public Health Observatory. Carr-Hill R., Chalmers-Dixon P. [citado 15 de Julio de 2013]. Disponible en: http://www.sepho.org.uk/extras/rch_handbook.aspx.

27. Costello AB, Osborne JW. Best practices in exploratory factor analysis: four recommendations for getting the most from your analysis. Pract Assess Res Eval. 2005;10:1-9.

28. Henson R.K., Roberts J., Kyle L. Use of Exploratory Factor Analysis in Published Research: Common Errors and Some Comment on Improved Practice. Educ Psychol Meas. 2006; 66:393-416.

29. División territorial de Barcelona. Departament Estadistica del Ajuntament de Barcelona. [citado 20 de Julio de 20013 . Dis ponible en: http://www.bcn.cat/estadistica/castella/terri/index. htm.
30. Smith GD, Hart C, Watt G, Hole D, Hawthorne V. Individual social class, area-based deprivation, cardiovascular disease risk factors, and mortality: the Renfrew and Paisley Study. J Epidemiol Community Health. 1998;52:399-405.

31. Havard S, Deguen S, Bodin J, Louis K, Laurent O, Bard D. A small-area index of socioeconomic deprivation to capture health inequalities in France. Soc Sci Med. 2008;67:2007-16.

32. Pickett KE, Pearl M. Multilevel analyses of neighbourhood socioeconomic context and health outcomes: a critical review. J Epidemiol Community Health. 2001;55:111-22

33. Regidor E, Gutierrez-Fisac JL, Banegas JR, Vicente D, Rodriguez-Artalejo F. Influencia a lo largo de la vida de las circunstancias socioeconómicas, de la inactividad física y de la obesidad sobre la presencia de síndrome metabólico. Rev Esp Salud Pública 2007; 81: 25-31*

34. Alvarez-Del Arco D, Llacer Gil de Ramales A, del Amo Valero J, Garcia-Fulgueiras A, Garcia-Pina R, Garcia-Ortuzar V et al. Metodología y logística de campo de un estudio multinivel sobre la influencia en Españade las características medioambientales en la salud mental de población autóctona y ecuatoriana inmigrante. Rev Esp Salud Pública. 2009;83:493-508.

35. Poza-Lara C, Fernández Cornejo JC. Una aproximación a la construcción de un indicador de pobreza multidimensional. ¿Cuáles son los focos de riesgo en España? Rev Mét Cuant Econ Emp. 2010; 10, 43-72. 